Die Bedeutung von Menschen mit Behinderungen für einen Betrieb - Kommentar aus Sicht einer

Schwerbehindertenvertretung

Alfons Adam

\title{
I. Problemaufriss
}

Das Thema „Älter werden in Betrieben“ wird die größte Herausforderung des nächsten Jahrzehnts sein. Die Erfahrung zeigt, dass ältere Menschen nicht öfter erkranken, wenn es aber geschieht, werden sie oft schwerbehindert. Die Schwerbehindertenvertretungen weisen schon seit vielen Jahren auf diese Herausforderung hin, wurden aber viele Jahre belächelt, und es wurde auf Seiten der Arbeitgeber das Gefühl entwickelt, als wenn sich alles von selbst erledige. Es wurden Frühpensionierungen und Altersteilzeiten zur Bewältigung dieser Herausforderung gewählt. In den Jahren hat man sehr viel wertvolle Zeit vertan, sich dem Thema in geeigneter Weise zu stellen.

\section{Das Daimler-Werk Bremen}

1. Zu der Entwicklung der schwerbehinderten Beschäftigten in den Betrieben am Beispiel des Daimler-Werks in Bremen

1993 lag die Schwerbehindertenquote bei 2,6 \% bei ca. 17000 Beschäftigten -2013 schon bei 7,6\% bei 12500 Beschäftigten. Diese Entwicklung zeigt sich in ähnlicher Weise in der ganzen Automobilindustrie und bei deren Zulieferern.

Positiv bleibt festzustellen, dass wir es in unserem Unternehmen geschafft haben, dem Thema „Ausbildung von schwerbehinderten Jugendlichen" ein besonderes Augenmerk zu widmen, und es ist uns in den letzten 8 Jahren gelungen, über 250 junge Menschen in eine Ausbildung bei Daimler zu bekommen und allen nach der Ausbildung ein festes Arbeitsverhältnis zu bieten. 
Darüber hinaus bieten wir pro Jahr ca. 40 jungen Menschen mit einer Lernbehinderung einen Ausbildungsplatz an.

\section{Steigende Zahl von Schwerbehinderten Menschen, besonders von Gleichgestellten}

Weit über $90 \%$ der Menschen sind während ihres Arbeitslebens schwerbehindert geworden. Gab es 1993 noch genügend leichte Tätigkeiten, wie z.B. in der Kontrolle, Logistik, Vormontage oder auch als Reiniger, Gärtner oder Waschraumwärter, sind diese Tätigkeiten unter dem wirtschaftlichen Druck fast vollständig fremdvergeben worden, und es fehlen die Arbeitsplätze für schwerbehinderte und ältere Kolleginnen und Kollegen.

Auch der Abschluss der Integrationsvereinbarung im Jahr 2003 hat die Situation nicht wesentlich verbessert. Aus diesem Grund besteht zurzeit nicht die Bereitschaft der Schwerbehindertenvertretung, in Gespräche zu einem Aktionsplan einzutreten, da letztendlich zu befürchten ist, dass viele Dinge, die in einen Aktionsplan zur betrieblichen Integration gehören, in der Realität nicht umgesetzt werden und dafür in Deutschland noch keine Kultur besteht. Auch an der Stelle müssen von der Politik klare Ansagen erwartet werden.

\section{Gesetzliche Instrumente und deren Bewertung im praktischen Prozess}

\section{Arbeitsschutzgesetz-Gefährdungsbeurteilung}

Das Arbeitsschutzgesetz und die Gefährdungsbeurteilung haben die Situation ebenfalls nicht entschärft. Allerdings ist ergonomisch sehr viel geschehen und hat die Arbeitsplätze positiv verändert. Eine besondere Situation stellt allerdings die Austaktung der Bänder in den Betrieben dar. Viele Kolleginnen und Kollegen können mit den Bandgeschwindigkeiten nicht mehr mithalten, und man erlebt es häufig, dass sich vorhandene Erkrankungen dadurch verschlimmern oder entstehen.

Ein großes Thema, an das sich viele noch nicht herantrauen, ist das Thema Barrierefreiheit. Die Barrierefreiheit sollte noch weitreichender als bisher im Arbeitsschutzgesetz geregelt sein. 


\section{Betriebliches Eingliederungsmanagement (BEM)}

Das BEM, richtig angewendet, ist ein unerlässliches Werkzeug zur Bewältigung unserer Herausforderungen. Leider erlebt man, dass das BEM von vielen Unternehmen nur BEM genannt wird und die Vorgehensweise unverändert zu früher ist, indem man weiter Krankenrückkehrgespräche mit mahnendem Charakter führt. Allerdings darf man auch den Aufwand bei einem richtig durchgeführten BEM nicht unterschätzen oder verschweigen. Dieses führt dazu, dass viele Arbeitgeber den Aufwand scheuen. In vielen Betriebsratsgremien ist der positive Gedanke zum BEM auch noch nicht angekommen, und viele Betriebsräte sind nicht bereit, sich auf eine Betriebsvereinbarung einzulassen oder sich am BEM zu beteiligen, da sie hinter dem BEM eine Maßnahme der Krankenverfolgung vermuten. Hier ist der Gesetzgeber gefordert, das Gesetz zu einem verpflichtenden BEM mit andern Rechtsfolgen bei Nichtbeachtung zu verändern.

\section{Fazit-Beschäftigung von Schwerbehinderten als Wettbewerbsnachteil?}

So lange die Gesetzeslage und die Vorschriften zur Beschäftigung von Schwerbehinderten Menschen so vage geregelt sind und sich fast $40 \%$ der deutschen Unternehmen durch eine Zahlung aus der Portokasse gänzlich von einer Beschäftigung befreien, ist dieses sehr wohl ein Wettbewerbsnachteil für Unternehmen die sich auf dem Gebiet engagieren. Wenn dann darüber hinaus die Fördermöglichkeiten sehr aufwendig beantragt werden müssen und die Kommunikation mit den Behörden so schwierig und Zeitaufwendig sind, muss man sich nicht wundern, dass Inklusion in den Betrieben nicht, oder nur sehr zaghaft stattfindet.

\section{Ausblick-Notwendigkeit anderer Gesetze?}

In der Tat sind gesetzliche Veränderungen im SGB nötig, insbesondere bedarf es eines proaktiven Gesetzes, das den Menschen hilft, und kein SGB, das Betroffene zum Spielball der Behörden und Leistungsträger macht. 
Für die Schwerbehindertenvertretungen, die Motor der Inklusion sind, müssen neue, zeitgemäße Regelungen im SGB IX geschaffen werden, z.B.:

- eine Unwirksamkeitsklausel für getroffene Entscheidungen ohne Anhörung der Schwerbehindertenvertretungen;

- klare Regelungen zu einem verpflichtenden BEM und der Integrationsvereinbarung und einem klar geregelten Vorgehen im BEM. Damit ist den Unternehmen und den Betroffenen gleichermaßen geholfen;

- die drastische Erhöhung der Ausgleichsabgabe für Unternehmen, die sich völlig der Beschäftigung entziehen;

- eine Ausweitung der Zuständigkeit für die Vermittlung von Menschen mit einer Behinderung auf die Integrationsämter;

- keine Ansiedelung der Bußgeldverfahren bei der Bundesagentur für Arbeit, da diese in einem Interessenskonflikt zu dem Vermittlungsauftrag steht, sondern eventuell, das wäre Vorstellbar, bei der Zollbehörde. 